\title{
IN-SITU FILM THICKNESS MEASUREMENTS FOR REAL-TIME MONITORING AND CONTROL OF ADVANCED PHOTORESIST TRACK COATING SYSTEMS
}

\author{
Thomas E. Metz, Richard N. Savage, Horace O. Simmons \\ SC Technology, 368 Earhart Way, Livermore, CA 94550
}

\begin{abstract}
This paper will explore the methodologies of real-time measurement of photoresist film thickness on silicon wafers using multi-wavelength reflection interferometry. Reflected light from the wafer's surface, containing the interference profile, is collected in-situ via a fiber optic cable and film thickness is determined in real-time via a pattern recognition algorithm. The instrumentation used to make this measurement and its application towards optimizing track performance during spin-coating and bake will be discussed.

Data demonstrating basic thickness versus spin-time and thickness versus bake-time profiles acquired on-line without process disruption will be presented along with its utilization towards minimizing process set-up and machine qualification. Moreover, the advantages of characterizing film thickness on-line and in real-time will be reviewed.
\end{abstract}

\section{INTRODUCTION}

Measuring each wafer after coating with Photoresist is becoming a necessity in the semiconductor industry's movement toward sub-micron linewidths. With uniformities of 20 Angstroms (total indicated range) being specified for waferto-wafer coatings, each process coating parameter (resist dispense rate and volume, spin speed accuracy, bake temperature and ambient humidity and temperature to name a few) must be optimized. On-line film thickness measurements can provide insights into characterizing and optimizing these parameters and provide statistical process control (SPC) measurements for each wafer run. Moreover, making measurements on-line and in real-time reduces track setup and process qualification time $(1,2)$. 


\section{METHOD}

Multi-wavelength reflection interferometry is employed to determine the thickness of the photoresist film coated on the surface of a silicon wafer. This technique is commonly used to measure film thickness values off-line at inspection stations ( 3 ), and has also been utilized to make thickness measurements in-situ during photoresist removal (4).

As illustrated in figure 1, the sample is illuminated with a quarty halogen lamp which is filtered so as not to expose the resist under measurement (Figure 1). A bifurcated fiber optic cable transports the illumination light through a lens assembly where it is focused or collimated to the wafer surface. The lens can be placed from 4 to 12 inches from the surface of the sample. The reflected light containing the interference profile is returned through the same lens and cable to a spectrometer.

FIGURE 1

\section{ON-LINE FILM THICKNESS MEASUREMENT}

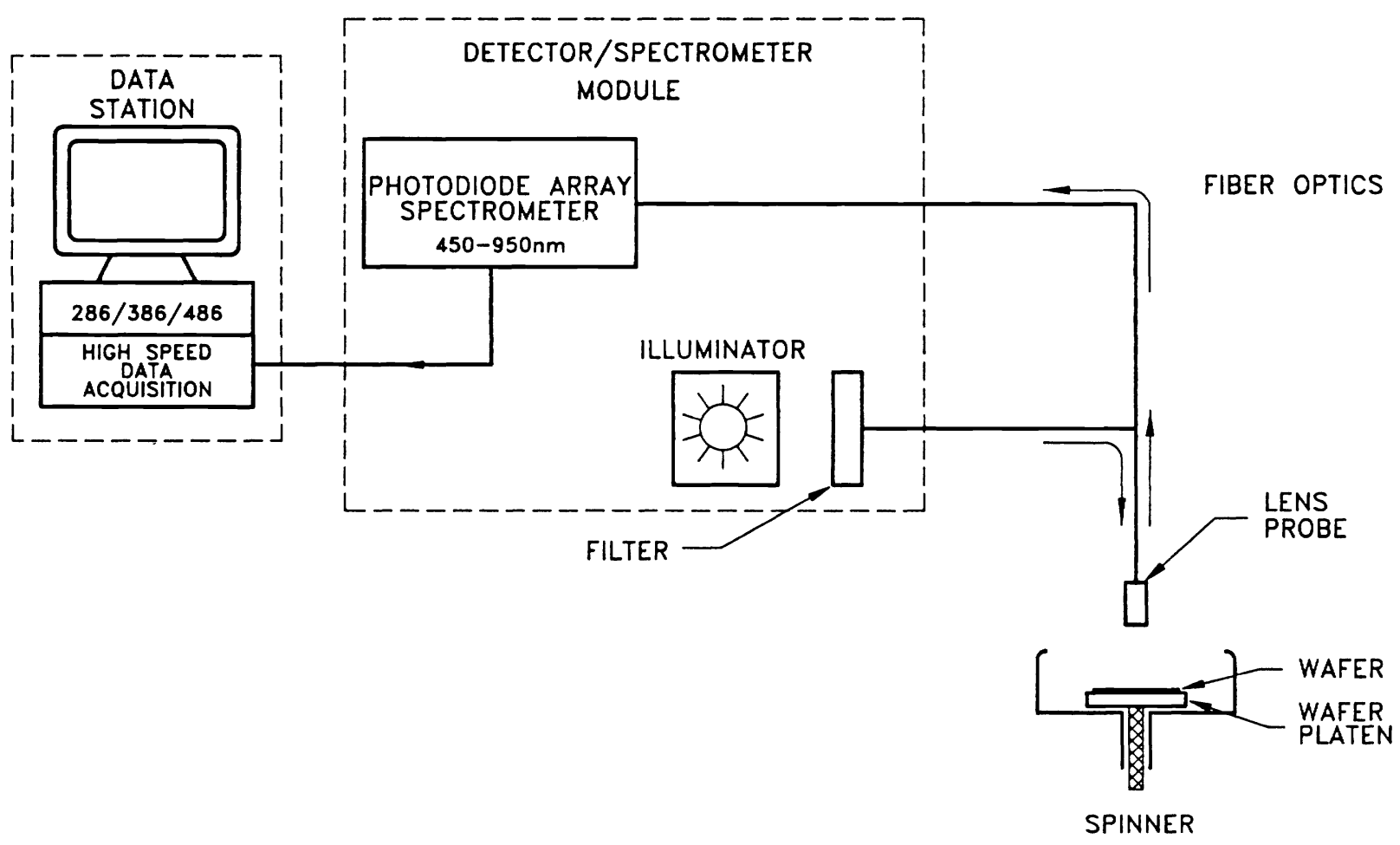


Speed was of the essence, so a spectrometer teamed up with a Photodiode Array was used rather than a scanning monochrometer to gather the light reflected from the sample. This method saves much time by reducing the spectrum acquisition time from seconds to milliseconds. The spectrometer is tuned to accept light in the 450 to $950 \mathrm{~nm}$ range. The signal from the $\mathrm{PDA}$ is inputed into a data acquisition $I / O$ board located in an AT type computer. The AT then processes this information using a pattern recognition software algorithm to compute the exact film thickness. (5) Using a more powerful 386 or 486 based machine speeds up the computing time allowing four to six measurements per second.

\section{EXPERIMENT}

The film thickness measurement system (SC Technology Model INS-800-1) was installed with the lens assembly mounted over a commercial resist spin coater module; thereby providing film thickness values during spin deposition. We observed in this case that the bulk of the resist was removed in the first 10 seconds, and very little resist was removed after 30 seconds (Figure 2). This data is probably not unknown to a lithography engineer, but real time monitoring enables the engineer to find the exact spin time needed to obtain the film thickness desired. Additionally, the slope of the change in thickness with spin time may be a good indication of the consistency of the spin rate.

RESULT: A reduction in spin time was implemented giving an increase in throughput.

The second experiment involved installing the measurement lens assembly over a bake station. The results are illustrated in Figure 3 ; notice that no resist thickness is lost until the wafer reaches the critical temperature causing desolvation. This occurs approximately five seconds into the bake cycle. A rapid solvent reduction then takes place and virtually no change happens after 49 seconds. We also observed changes in this curve when resist viscosities were changed.

RESULT: A reduction in bake time.

During the third experiment thickness values were measured over a chill plate, after spin and bake had taken place, to see if the film thickness was within the targeted specification. A go/no-go parameter could be set up providing an on-line $Q C$ check with no operator interaction 
FIGURE 2

\section{Photoresist Thickness vs. Spin Time 8-inch Wafer on MTI Flexi Fab}

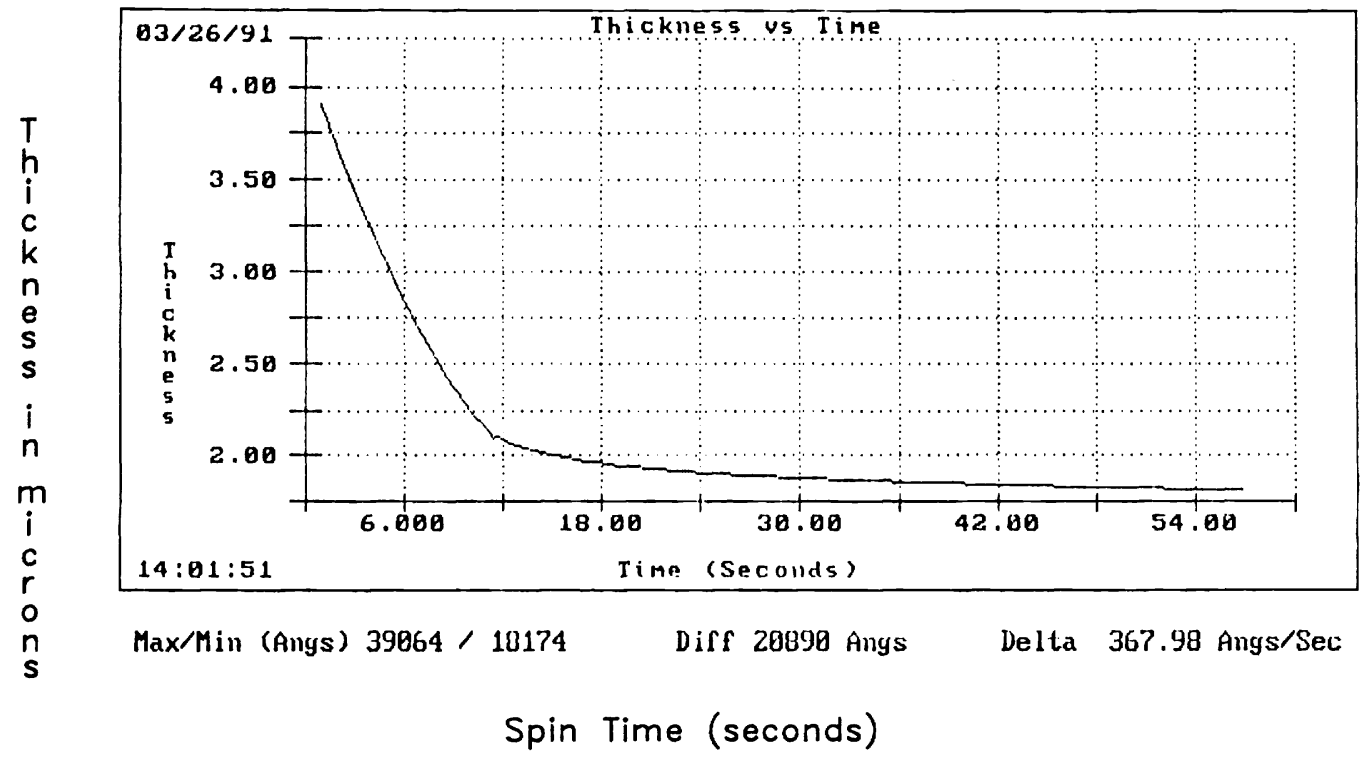

FIGURE 3

\section{Photoresist Thickness vs. Bake Time 8-inch Wafer on MTI Flexi Fab}

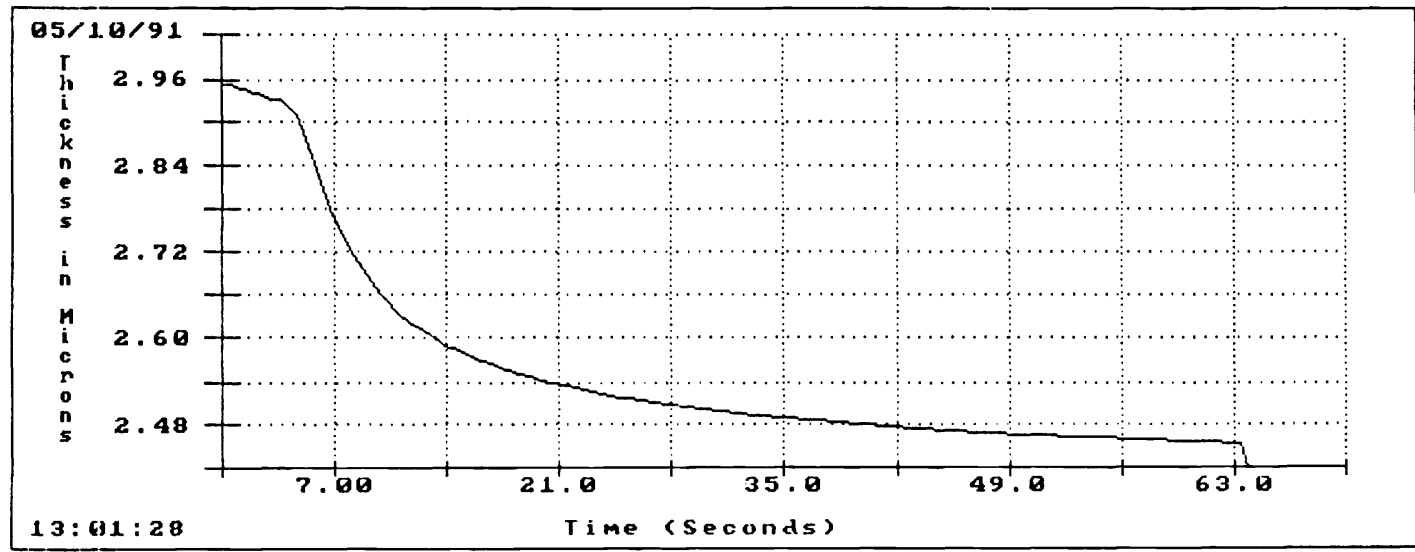

Bake Time (seconds) 
required. Measuring at the chill plate before the wafers were loaded into a cassette eliminated the need to take the wafers off-line to a table top measuring system thereby saving time, wafer handling and transport, and possible mix ups.

RESULT: An increase in productivity.

Finally, the lens assembly was attached to a support arm capable of rotary motion. The arm moved the lens assembly across the diameter of the wafer while measurements were made. This gave us a uniformity profile of the resist thickness as shown in Figures 4 and 5 . We were able to see dishes and domes of photoresist across the wafer. While these measurements were made off-line, it would be conceivable to mount the lens assembly above the chill plate allowing these measurements to be made on-line. This would enable the process engineer to rapidly make adjustments to the spin speed, photoresist dispense volume, photoresist viscosity, or other parameters to correct for the dish/dome problems without taking the wafer off-line to a measurement station.

RESULT: Faster set-up and qualification time.

\section{CONCLUSION}

On-line film thickness monitoring done in real-time has shown to be able to speed up spin and bake optimization. This method can also be used to detect equipment problems such as dispense variations, and inadequate bake times. Uniformities across each wafer and from lot-to-lot can be rapidly monitored and the information used for statistical process control analysis. Additionally, variations outside of a preset tolerance can be automatically detected and defective coatings can be renoved from the track for rework.

\section{ACKNOWLEDGMENTS}

The authors wish to thank william Liggett, Rachel Trujillo, and Bob J. Williams of TFI TELEMARK for the use of their MTI FLEXI FAB track system, for suppling wafers, and for assistance in operating the track. 
FIGURE 4

\section{Photoresist Thickness vs. Position} across Diameter of a 6-inch Wafer

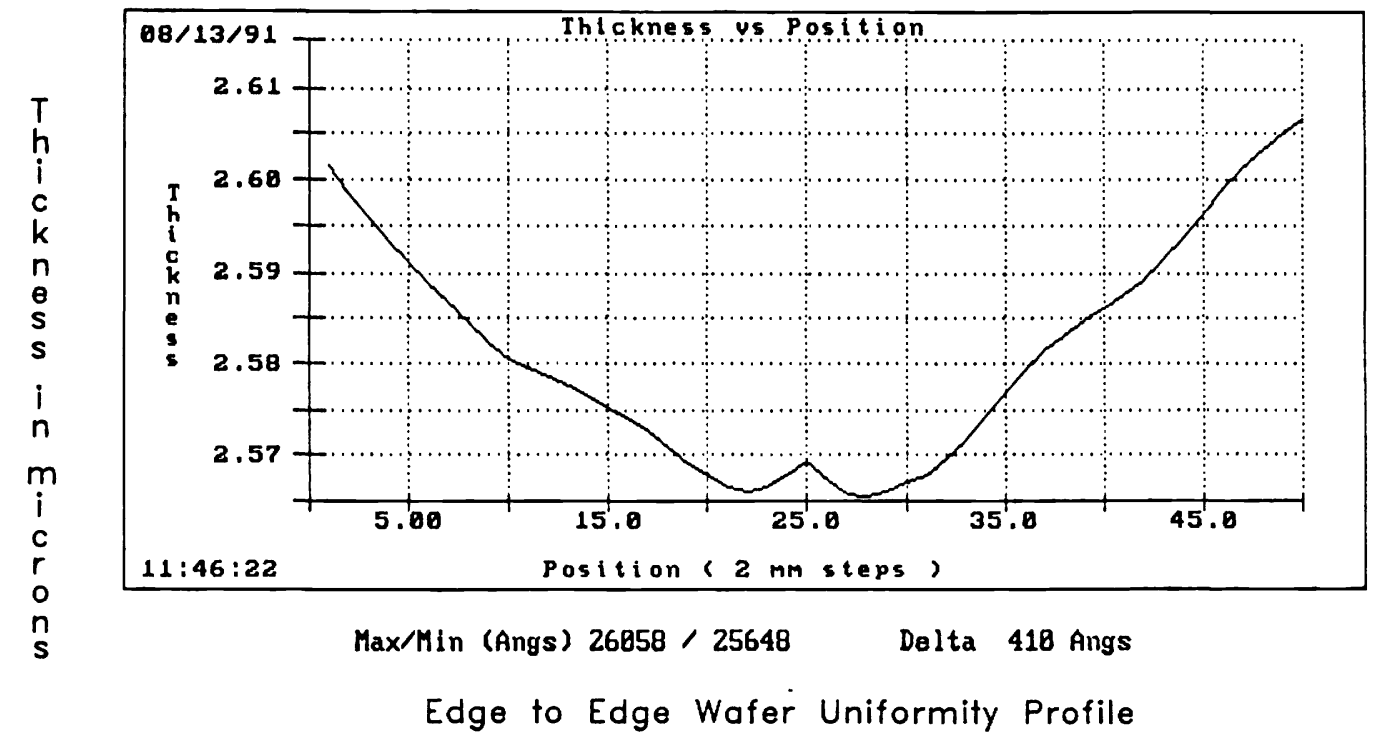

FIGURE 5

\section{Photoresist Thickness vs. Position across Diameter of a 6-inch Wafer}

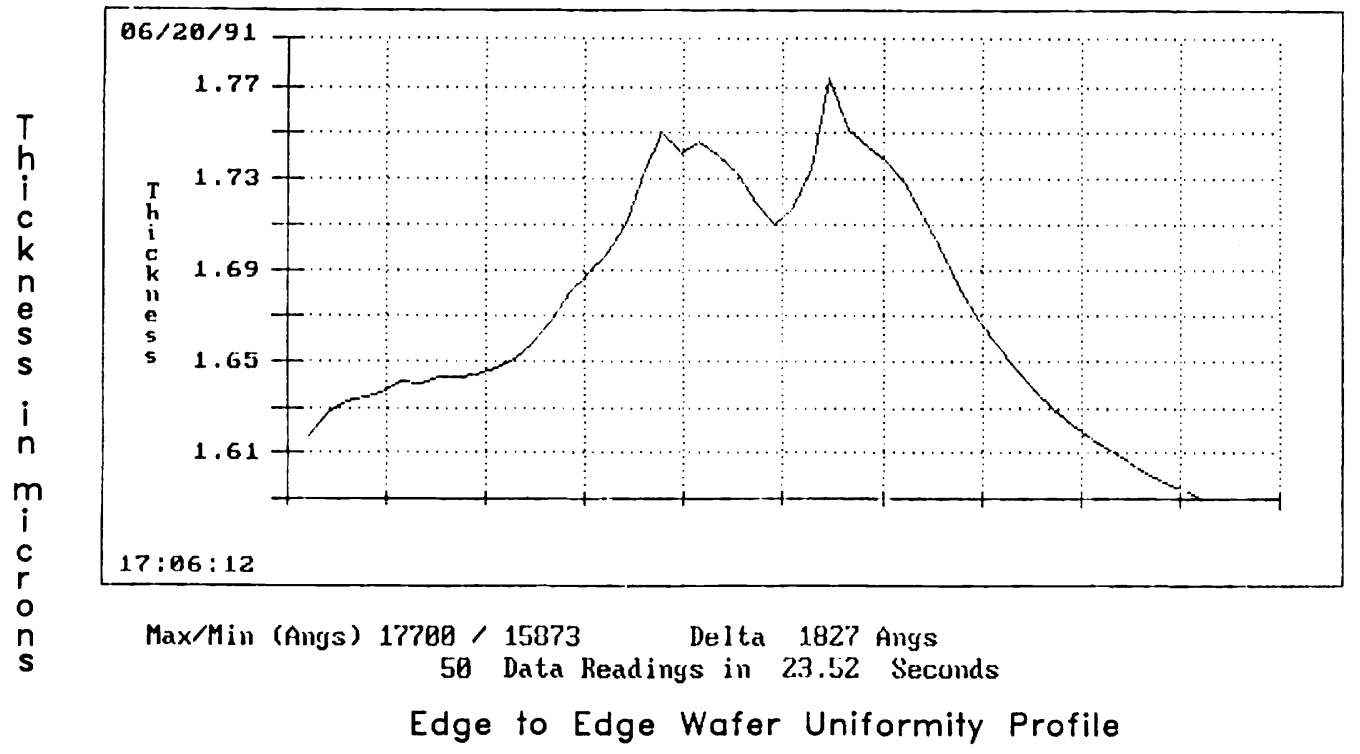




\section{REFERENCES}

1. K.M. Sautter, G.H. Memovitch, T. Batchelder; "In-line Photoresist thickness Measurement for Sub-Micron Process Control," Microelectronics Manufacturing and Test, September, 1987.

2. B.Mohondro, "Photoresist Application: An Art and a Science," Microelectronic Manufacturing and Test, April 1990

3. W.A. Keenan, M.E. Keefer, P.E. Gise and L.A. Thornquist, "Film Thickness Mapping of Advanced Wafer Production Process," Microelectronic Manufacturing and Technology, February 1991

4. J.T. Davies, T.E. Metz, R.N. Savage and H.O. Simmons, "Real time in-situ measurement of film thickness and uniformity during plasma ashing of photoresist," SPIE Vol 1392, Advanced Techniques for Integrated Circuit Processing $1990, \mathrm{Pg} 551$.

5. R.N. Savage, "Real Time In-Situ Measurements of film Thickness and Uniformity During Deposition and Etch Processes," Society of Vacuum Coaters, 34 th Annual Technical Conference, Philadelphia, PA, March 1991 